\title{
Relativistic Quantum Mechanics on the $\mathrm{SL}(2, \mathrm{R})$ Spacetime
}

\author{
T. Fülöp* \\ Institute for Theoretical Physics \\ Eötvös University, Budapest, Hungary \\ September 10, 1996
}

\begin{abstract}
The Schrödinger-type formalism of the Klein-Gordon quantum mechanics is adapted for the case of the $S L(2, \mathbf{R})$ spacetime. The free particle case is solved, the results of a recent work are reproduced while all the other, topologically nontrivial solutions and the antiparticle modes are also found, and a deeper insight into the physical content of the theory is given.
\end{abstract}

\section{Introduction}

Recently the classical and quantum mechanics of the zero spin particle moving freely on the $S L(2, \mathbf{R})$ group manifold were examined in [1]. The theory of the system was constructed via Hamiltonian reduction, a method becoming increasingly popular nowadays in the field of $\mathcal{W}$-algebras and integrable models (for references see [1]). This problem is of interest because of a number of aspects. From the point of view of conformal field theory this system is the point particle analogue of the $S L(2, \mathbf{R})$ WZNW model, having an analogous reparametrization invariance property. From the aspect of string theory the system can be considered to describe the 'centre-ofmass motion' of a (WZNW) string on the $S L(2, \mathbf{R})$ spacetime. The problem is also of interest from the point of view of general relativity because it offers an example of an exactly solvable quantum system on a curved spacetime background.

After constructing the classical theory, the authors in [1] quantize the system by considering unitary, irreducible representations of the algebra formed by the symmetry currents corresponding to the left and right translation invariance. The results set some interesting problems and questions. One of them is that, due to the representations given in [1], the value of the mass of the particle cannot

*E-mail: fulopt@hal9000.elte.hu 
be arbitrary. Only a discrete series is allowed for the possible mass values. It is plausible to conjecture that this condition is of topological origin. The topology of the $S L(2, \mathbf{R})$ group manifold is $\mathbf{R}^{2} \times \mathcal{S}^{1}$, and the presence of a compact dimension would be responsible for the mass quantization condition. Then we may ask whether this condition is necessary for a consistent solution or the other mass values also correspond to additional - consistent, while topologically 'nontrivial' - solutions. The idea is that if the condition holds then the wave function of the particle is singlevalued around the $\mathcal{S}^{1}$ direction, while for other mass values a nontrivial constant phase factor would be present.

Another question is that one expects the appearance of antiparticle modes, similarly as in the case of Minkowski spacetime. It would be nice to find them, too, as a natural part of the complete space of states. Thirdly, in the usual cases of nonrelativistic quantum mechanics or quantum mechanics on Minkowski spacetime the state space, the Hamiltonian, etc. of a quantum system suppose that initially an inertial reference frame has been chosen. What is the corresponding step in the case of the $S L(2, \mathbf{R})$ spacetime? Special attention has to be payed to this problem as the spacetime in question is a curved one.

Finally, it would be interesting to answer some other questions concerning the formalism, including how to define observables and expectation values or how to handle the case when an external field is present.

In this paper these questions will be answered. Our method is the adaptation of the Schrödinger-type formalism of the special relativistic Klein-Gordon quantum mechanics to the case of the $S L(2, \mathbf{R})$ spacetime. The advantage of using this extension of the Klein-Gordon framework is that in this way the physical properties of the system become more transparent. This formalism makes the particle and antiparticle modes and the corresponding charge symmetry more visible, and provides the theory a consistent physical interpretation (including, e.g., the definition of observables and expectation values).

The paper is organized as follows: in Sec. II the results of [1] are presented. The necessary ingredients of the formalism of the quantum mechanics of the spin zero particle on Minkowski spacetime are summarized in Sec. III. The properties of the $S L(2, \mathbf{R})$ spacetime and the choice of a suitable reference frame on it are discussed in Sec. IV. In Sec. V the formalism presented in Sec. III is set on the $S L(2, \mathbf{R})$

spacetime. The resulting theory is solved in the free particle case in Sec. VI. The left and right symmetric aspects of the free system are discussed in Sec. VII.

\section{Quantization via Hamiltonian reduction}

$S L(2, \mathbf{R})$ is a three dimensional Lie group, the naturally arising metric is

$$
h_{\mu \nu}(x)=\frac{1}{2} \operatorname{Tr}\left[g^{-1} \partial_{\mu} g g^{-1} \partial_{\nu} g\right]
$$


(with a local parametrization $x \mapsto g(x) \in S L(2, \mathbf{R})$ ). This smooth metric is nondegenerate and proves to be of Lorentz signature, thus $S L(2, \mathbf{R})$ can be considered as a $2+1$ dimensional curved spacetime.

The classical and the quantum theory of a free particle on the $S L(2, \mathbf{R})$ spacetime is constructed in [1] as follows. Classically the action

$$
I_{0}=-\kappa \int d t \sqrt{h_{\mu \nu}(x) \dot{x}^{\mu} \dot{x}^{\nu}}
$$

describes a particle of mass $\kappa>0$, with $x^{\mu}(t)$ denoting the trajectory of the particle. The action $I_{0}$ can be obtained from a more appropriate quadratic action $I$ by imposing a given constraint. This first class constraint arises as a consistency condition on the canonical momenta of the system $I$, and the local gauge symmetry it generates is nothing but the reparametrization invariance of the system. Then, according to the method of Hamiltonian reduction, the reduced phase space can be obtained by factorizing the constrained surface with respect to the gauge symmetry.

In addition to the reparametrization invariance the system is invariant under the left and right transformations $g \mapsto f g, g \mapsto g \tilde{f}^{-1}, f, \tilde{f} \in S L(2, \mathbf{R})$. The reduced phase space is found to be of the form $\mathcal{O}_{K} \times \mathcal{O}_{-K}$ where $\mathcal{O}_{K}$ and $\mathcal{O}_{-K}$ are the coadjoint orbits of the left, resp. the right, transformations passing through a fixed timelike vector $K$ arbitrarily chosen from $\operatorname{sl}(2, \mathbf{R})$, the Lie algebra of $S L(2, \mathbf{R})$. The left and right symmetry currents parametrize the reduced phase space and form two independent $\operatorname{sl}(2, \mathbf{R})$ algebras under the Poisson brackets.

The quantum theory is obtained in [1] via quantizing the reduced phase space. Unitary, irreducible representations of the algebra $\operatorname{sl}(2, \mathbf{R})$ formed by the symmetry currents on $\mathcal{O}_{K}$ and $\mathcal{O}_{-K}$ are sought. The state space is spanned by the tensorial product of two such representations, which have to share the same value for the Casimir due to a mass shell condition. Such a state (denoted by $\left.\left|n_{L}, n_{R}\right\rangle\right)$ is labelled by two non-negative integers. The quantum commutation relations for the left current in an appropriate basis are

$$
\left[L_{0}, L_{1}\right]=-2 i L_{2}, \quad\left[L_{1}, L_{2}\right]=2 i L_{0}, \quad\left[L_{2}, L_{0}\right]=-2 i L_{1} .
$$

With $L_{ \pm}=L_{1} \mp i L_{2}$ one finds

$$
\begin{aligned}
L_{0}\left|n_{L}, n_{R}\right\rangle & =2\left(n_{L}+j\right)\left|n_{L}, n_{R}\right\rangle, \\
L_{+}\left|n_{L}, n_{R}\right\rangle & =2 \sqrt{\left(n_{L}+2 j\right)\left(n_{L}+1\right)}\left|n_{L}+1, n_{R}\right\rangle, \\
L_{-}\left|n_{L}, n_{R}\right\rangle & =2 \sqrt{\left(n_{L}-1+2 j\right) n_{L}}\left|n_{L}-1, n_{R}\right\rangle .
\end{aligned}
$$

In a similar manner, the action of the right current on the states is

$$
\begin{aligned}
R_{0}\left|n_{L}, n_{R}\right\rangle & =-2\left(n_{R}+j\right)\left|n_{L}, n_{R}\right\rangle, \\
R_{-}\left|n_{L}, n_{R}\right\rangle & =-2 \sqrt{\left(n_{R}+2 j\right)\left(n_{R}+1\right)}\left|n_{L}, n_{R}+1\right\rangle, \\
R_{+}\left|n_{L}, n_{R}\right\rangle & =-2 \sqrt{\left(n_{R}-1+2 j\right) n_{R}}\left|n_{L}, n_{R}-1\right\rangle,
\end{aligned}
$$


where $j=\frac{1}{2}\left(1+\sqrt{1+\kappa^{2}}\right)$. As in [1] the left and right representations are chosen from the discrete series $D_{j}^{ \pm}[2], j$ has to take one of the values $\frac{3}{2}, \frac{4}{2}, \frac{5}{2}, \ldots$ Consequently, the mass of the particle is not arbitrary but must come from a discrete series of allowed values.

The states $\left|n_{L}, n_{R}\right\rangle$ are eigenstates of the energy and the angular momentum, which operators can be identified as $\frac{1}{2}\left(L_{0}-R_{0}\right)$ resp. $\frac{1}{2}\left(L_{0}+R_{0}\right)$, the corresponding eigenvalues are $n_{L}+n_{R}+2 j$ resp. $n_{L}-n_{R}$. The energy levels are positive definite and spaced integrally, the angular momentum takes integer values.

\section{The quantum mechanics of the zero spin par- ticle on Minkowski spacetime}

To recall the basic elements of the Schrödinger-type formalism of the quantum mechanics of the zero spin particle on Minkowski spacetime we follow the approach of Feshbach and Villars [3]. This formalism is the close analogy of the one of the spin $\frac{1}{2}$ particle case and gives a consistent and well-interpretable one-particle quantum theory.

We start from the Klein-Gordon equation for a particle with mass $\kappa$ and electric charge $e$ in the presence of an electromagnetic potential $A^{\mu}=\left(A^{0}, A^{k}\right)=\left(A^{0}, \boldsymbol{A}\right)$ :

$$
\left(D^{\mu} D_{\mu}-\kappa^{2}\right) \psi=0
$$

where $D_{\mu}=\frac{\partial}{\partial x^{\mu}}-i e A_{\mu}$ (we work in $\hbar=c=1$ ). Our purpose is to reformulate (6) as an equation of the form $i(\partial \Psi / \partial t)=H \Psi$ with an appropriate $\Psi$. This can be achieved by considering $D_{0} \psi$ as an independent degree of freedom and introducing the two-component column vector $\Psi$ with components $\psi$ and $D_{0} \psi$. More precisely, later convenience suggests to define $\Psi$ as

$$
\Psi=\left(\begin{array}{c}
\varphi \\
\chi
\end{array}\right)=\frac{1}{\sqrt{2}}\left(\begin{array}{c}
\psi+\frac{i}{\kappa} D_{0} \psi \\
\psi-\frac{i}{\kappa} D_{0} \psi
\end{array}\right) .
$$

Then (6) can be rewritten as

$$
\begin{aligned}
i(\partial \varphi / \partial t) & =\frac{1}{2 \kappa}\left(\frac{1}{i} \boldsymbol{\nabla}-e \boldsymbol{A}\right)^{2}(\varphi+\chi)+\left(\kappa-e A_{0}\right) \varphi \\
i(\partial \chi / \partial t) & =-\frac{1}{2 \kappa}\left(\frac{1}{i} \nabla-e \boldsymbol{A}\right)^{2}(\varphi+\chi)-\left(\kappa+e A_{0}\right) \chi
\end{aligned}
$$

the operator $H$ can be read off from (8).

From (6) the current four-vector defined as $j_{\mu}=$ const. $\left(\psi^{*} D_{\mu} \psi-\right.$ c.c. $)$ proves to be conserved. One can express $j_{\mu}$ with $\Psi$ as well, for example the density reads $j_{0}=\Psi^{*} \sigma_{3} \Psi=\varphi^{*} \varphi-\chi^{*} \chi\left(\sigma_{k}\right.$ denotes the Pauli matrices $)$. The density $j_{0}$ is not positive definite, $j_{\mu}$ is interpreted not as the probability current but as the charge 
current. If $\Psi$ satisfies the equation $i(\partial \Psi / \partial t)=H(e) \Psi$ then the charge conjugate wave function

$$
\Psi_{c}=\left(\begin{array}{c}
\chi^{*} \\
\varphi^{*}
\end{array}\right)
$$

satisfies $i\left(\partial \Psi_{c} / \partial t\right)=H(-e) \Psi_{c}$ (where $H(-e)$ differs from $H(e)$ only by the sign of the electric charge), the density corresponding to $\Psi_{c}$ is $\Psi_{c}^{*} \sigma_{3} \Psi_{c}=-\Psi^{*} \sigma_{3} \Psi$. Thus we can see that the theory actually describes two degrees of freedom with opposite charges (a particle and an antiparticle) and has a fundamental charge symmetry. The advantage of using $\varphi$ and $\chi$ as the two components of the wave function is that this property becomes apparent. In the nonrelativistic limit the two degrees of freedom decouple and lead to two independent Schrödinger equations of the usual form.

If $\int \Psi^{*} \sigma_{3} \Psi d^{3} x$ is positive/negative then let $\Psi$ be called 'positive', resp. 'negative', and be normalized so that this integral be +1 resp. -1 , expressing that the charge of such a state is $+e$ resp. $-e$.

The inner product that turns out to be appropriate for this formalism is

$$
\left(\Psi_{1}, \Psi_{2}\right)=\int \Psi_{1}^{*} \sigma_{3} \Psi_{2} d^{3} x=\int\left(\varphi_{1}^{*} \varphi_{2}-\chi_{1}^{*} \chi_{2}\right) d^{3} x
$$

This inner product is not positive definite, the space of the wave functions is not a Hilbert space, on the contrary to the nonrelativistic case. Physical quantities correspond to Hermitian operators acting on the $\Psi$-s where Hermiticity, the expectation value of an operator and such notions are defined with respect to the inner product (10). An interesting consequence of the indefiniteness of the inner product is that the eigenvalues of an operator are not necessarily expectation values as well. For example, if $A_{\mu}=0$ then the expectation value of the Hamiltonian in an eigenstate with eigenvalue $E$ is $|E|>0$. Similarly, the expectation value of the kinetic energy operator of the general case $A_{\mu} \neq 0$ always proves to be positive definite.

We remark that further interpretation issues and the case of the neutral particles are also discussed in [3].

\section{The $\mathrm{SL}(2, \mathrm{R})$ spacetime}

The Schrödinger-type formalism of the Klein-Gordon quantum mechanics required the choice of an inertial reference frame on the Minkowski spacetime. As we want to adapt this formalism for the $S L(2, \mathbf{R})$ spacetime, we have to solve the nontrivial problem of finding an analogous step on the curved spacetime of $S L(2, \mathbf{R})$.

Let us consider the choice of a reference frame on the Minkowski spacetime the following way. We start by choosing a timelike vector field on the spacetime. Then we construct spacelike hypersurfaces being orthogonal to the integral curves corresponding to this vector field. The time coordinate is introduced as the parameter along the integral curves and the space coordinates are introduced to parametrize the 
hypersurfaces. An inertial reference frame is such a special reference frame that the vector field chosen is constant, the same timelike vector (absolute or four-velocity) is attached to each spacetime point, the integral curves are parallel straight lines and the orthogonal hypersurfaces are parallel hyperplanes. In other words, we produce an inertial reference frame by considering an absolute velocity vector in a spacetime point and then we shift this timelike vector by a parallel translation to all the other spacetime points.

It is this translation idea that is appropriate to define the analogue of an inertial reference frame on $S L(2, \mathbf{R})$. Let us choose its identity element as one of the spacetime points and let us consider a timelike vector $U$ in its tangent space, i.e., in $\operatorname{sl}(2, \mathbf{R})$. Then we shift this vector to the tangent space of a spacetime point $g$ by the natural translation $g U$ (where this multiplication means simply the multiplication of matrices). However, an important difference from the Minkowski case is that now the left and the right translation are not equal, $g U \neq U g$, as a consequence of the noncommutativity of the group $S L(2, \mathbf{R})$. Thus we can define a "left-inertial reference frame" and a "right-inertial reference frame" corresponding to the two possibilities of translation. Moreover, not only $g U$ and $U g$ can be chosen naturally, but also the average $\frac{1}{2}(g U+U g)$ (the "middle-inertial reference frame"). Straightforward calculations show that for each of these three timelike Killing vector fields the metric (看) is time-independent. The middle-inertial reference frame has an additional advantageous property: the space-time mixed components of $h_{\mu \nu}$ prove to be zero. That is why we choose the middle-inertial reference frame for the following considerations.

Now let us make use of the fact that, for a fixed $U$, a $g \in S L(2, \mathbf{R})$ can be given in the form $e^{\frac{1}{2} t U} e^{C} e^{\frac{1}{2} t U}$ where $t \in[0,2 \pi)$ and $C \in \operatorname{sl}(2, \mathbf{R}), C$ is orthogonal to $U$; any such $t$ and $C$ uniquely characterizes an element of $S L(2, \mathbf{R})$. (This statement can be proven with the aid of the formula $e^{\xi^{k} \sigma_{k}}=\cosh R \mathbf{1}+\sinh R / R \xi^{k} \sigma_{k}, R^{2}=\xi^{k} \xi^{k}$.) Accordingly, $\partial_{t} g=\frac{1}{2}(g U+U g)(C$ is kept fixed $)$. Thus by a coordinatization $C=C\left(x^{1}, x^{2}\right)$ and with $x^{0}:=t$ we obtain a middle-inertial coordinate system corresponding to $U$. For example, in polar coordinates: $x^{1}=r, x^{2}=\vartheta, C=$ $r \cos \vartheta X+r \sin \vartheta Y$ [with $X$ and $Y$ fixed such that $(U, X, Y)$ is an orthonormal basis in $\operatorname{sl}(2, \mathbf{R})]$ the metric tensor reads

$$
\left\{h_{\mu \nu}\right\}=\left(\begin{array}{ccc}
-\cosh ^{2} r & 0 & 0 \\
0 & 1 & 0 \\
0 & 0 & \sinh ^{2} r
\end{array}\right) .
$$

Remarkably, the topology of $S L(2, \mathbf{R})$ is $\mathbf{R}^{2} \times \mathcal{S}^{1}$ - the timelike geodesics are the closed ones-, hence this manifold cannot be covered with a single open coordinate patch. However, a middle-inertial coordinate system covers the whole $S L(2, \mathbf{R})$ if we identify $t=2 \pi$ with $t=0$. This way we can avoid the use of multiple patches.

We mention that the elements of $S L(2, \mathbf{R})$ can be given also in the form $e^{\xi X} e^{\eta Y} e^{\tau U}$, where $\xi, \eta \in \mathbf{R}$ and $\tau \in[0,2 \pi)$. Later we will make use of this fact, too. 


\section{Relativistic quantum mechanics on the $\mathrm{SL}(2$, R) spacetime}

To build up the relativistic quantum theory on the $S L(2, \mathbf{R})$ spacetime we follow the steps of Sec. III. Now the Klein-Gordon equation reads

$$
\left(\tilde{D}^{\mu} \tilde{D}_{\mu}-\kappa^{2}\right) \psi=0
$$

where $\tilde{D}_{\mu}=\nabla_{\mu}-i e A_{\mu}\left(\nabla_{\mu}\right.$ denotes the covariant derivative $)$. In a middle-inertial coordinate system the metric is time independent and its space-time mixed components are zero, thus re-writing (12) as a first order equation in the variable $\Psi$ introduced as in the flat case (cf. (7),$\left.D_{\mu}=\partial_{\mu}-i e A_{\mu}\right)$ yields

$$
\begin{array}{r}
i(\partial \varphi / \partial t)=\frac{1}{2 \kappa} \frac{1}{h^{00} \sqrt{-h}} D_{j}\left(\sqrt{-h} h^{j k} D_{k}\right)(\varphi+\chi) \\
+\frac{\kappa}{2}\left[\left(1-\frac{1}{h^{00}}\right) \varphi-\left(1+\frac{1}{h^{00}}\right) \chi\right]-e A_{0} \varphi, \\
i(\partial \chi / \partial t)=-\frac{1}{2 \kappa} \frac{1}{h^{00} \sqrt{-h}} D_{j}\left(\sqrt{-h} h^{j k} D_{k}\right)(\varphi+\chi) \\
+\frac{\kappa}{2}\left[\left(1+\frac{1}{h^{00}}\right) \varphi+\left(\frac{1}{h^{00}}-1\right) \chi\right]-e A_{0} \chi
\end{array}
$$

where $h$ is the determinant of the metric, $j, k=1,2$. The corresponding Hamiltonian can be read off from (13).

The inner product to be introduced as the analogue of the special relativistic one must satisfy some requirements, i.e., to be invariant under space $\rightarrow$ space transformations $\left(x^{1}, x^{2}\right) \rightarrow\left(x^{1^{\prime}}, x^{2^{\prime}}\right)$ and to ensure that the Hamiltonian be symmetric and the charge be conserved. The result is the appearance of a weight function in the integral (10):

$$
\left(\Psi_{1}, \Psi_{2}\right)=\int \Psi_{1}^{*} \sigma_{3} \Psi_{2} \sqrt{-h}\left(-h^{00}\right) d^{2} x=\int\left(\varphi_{1}^{*} \varphi_{2}-\chi_{1}^{*} \chi_{2}\right) \sqrt{-h}\left(-h^{00}\right) d^{2} x .
$$

It can be checked easily that the fundamental charge symmetry keeps valid without any modifications of the formulae of the Minkowski case. Similarly, the definition of observables and expectation values, as well as all other interpretation issues, also can be adapted appropriately from the special relativistic formalism.

We mention that the considerations of this section are applicable not only for the $S L(2, \mathbf{R})$ spacetime but, more generally, for any static spacetime, i.e., for such curved spacetimes that have a time independent metric with zero space-time mixed components (in a suitable coordinatization).

\section{The free masspoint}

Now let us solve the eigenvalue problem for the Hamiltonian of the free system. The free Hamiltonian is time independent-following from the time independence 
of the metric-, thus $H \Psi=E \Psi$ implies $\Psi(t)=\exp (-i E t) \Psi(0)$ (in the following this time dependence will always be understood to the eigenfunctions). Thus $\varphi(t)=\exp (-i E t) \varphi(0)$ and $\chi(t)=\exp (-i E t) \chi(0)$, and, consequently, $\psi(t)=$ $\exp (-i E t) \psi(0)$. As a result, an eigenfunction $\Psi$ can be expressed by means of $\psi$ only (!), from (77) one finds

$$
\Psi=\frac{1}{\sqrt{2}}\left(\begin{array}{c}
1+\frac{E}{\kappa} \\
1-\frac{E}{\kappa}
\end{array}\right) \psi=\left(\begin{array}{c}
\frac{1}{\sqrt{2}}\left(1+\frac{E}{\kappa}\right) \psi \\
\frac{1}{\sqrt{2}}\left(1-\frac{E}{\kappa}\right) \psi
\end{array}\right) .
$$

The inner product of a $\Psi_{1}$ with eigenvalue $E_{1}$ and a $\Psi_{2}$ with eigenvalue $E_{2}$ can also be expressed with the corresponding $\psi_{1}$ and $\psi_{2}$ :

$$
\left(\Psi_{1}, \Psi_{2}\right)=\frac{E_{1}+E_{2}}{\kappa} \int \psi_{1}^{*} \psi_{2} \sqrt{-h}\left(-h^{00}\right) d^{2} x
$$

Substituting the connection between $\Psi$ and $\psi$ into $H \Psi=E \Psi$ and working in polar coordinates - an appropriate concrete space coordinatization, cf. (11]) - gives

$$
\partial_{r}^{2} \psi+2 \operatorname{coth} 2 r \partial_{r} \psi+\frac{1}{\sinh ^{2} r} \partial_{\vartheta}^{2} \psi+\frac{E^{2}}{\cosh ^{2} r} \psi-\kappa^{2} \psi=0 .
$$

We can expand $\psi$ into Fourier series in the variable $\vartheta \in[0,2 \pi)$, thus expressing it as a linear combination of the functions $\exp (i m \vartheta), m \in \mathbf{Z}$. A term of this series is of the form

$$
\psi_{m, E}=W_{m, E}(r) e^{i m \vartheta} e^{-i E t},
$$

the corresponding $\Psi_{m, E}$ is an eigenfunction of the angular momentum operator $J=$ $-i \partial_{\vartheta}$ as well, hence determining all the $\Psi_{m, E^{-S}}$ means the common diagonalization of $H$ and $J$.

Eq. (17) implies an ordinary differential equation on $W_{m, E}$. This equation can be turned into the hipergeometric equation $z(1-z) d^{2} w / d z^{2}+[c-(a+b+1) z] d w / d z-$ $a b w=0$ in the new variable $z=\tanh ^{2} r$ and with $a=j+(|E|+|m|) / 2, b=$ $j+(-|E|+|m|) / 2, c=1+|m|, w=z^{(1-c) / 2}(1-z)^{(c-a-b-1) / 2} W_{m, E}$ where $j=\frac{1}{2}(1+$ $\sqrt{1+\kappa^{2}}$ ) (for the conventions and properties used concerning the hipergeometric equation see [4] or [5]). A solution of the hipergeometric equation (with fixed $a, b$ and $c)$ is the hipergeometric function $F(z) \equiv F(a, b ; c ; z)$ 伅. We choose

$$
G(z) \equiv G(a, b ; c ; z)=F(z) \int_{1}^{z} \frac{d \zeta}{\zeta^{c}(1-\zeta)^{a+b-c+1} F(\zeta)^{2}}
$$

as a linearly independent solution [7], the other solutions are linear combinations of $F$ and $G$. $F$ and $G$ are regular on $(0,1)$, in spite of eventual nodes of $F$.

Now let us search for a maximal orthogonal system of the eigenfunctions. $\Psi_{m_{1}, E_{1}}$ and $\Psi_{m_{2}, E_{2}}$ are orthogonal if $m_{1} \neq m_{2}$ because of their $\vartheta$-dependence. Thus it is enough to examine the case $m_{1}=m_{2}=m$. Then $\left(\Psi_{m, E_{1}}, \Psi_{m, E_{2}}\right)$, expressing with the corresponding $w_{1}$ and $w_{2}$, is

$$
\text { const. } \int_{0}^{1} z^{c-1}(1-z)^{a_{1}+b_{1}-c} w_{1}^{*} w_{2} d z
$$


(now $a_{1}+b_{1}=a_{2}+b_{2}$ and $c_{1}=c_{2}=c$ ). With the aid of the hipergeometric equation it is not hard to prove that

$$
\int_{p}^{q} z^{c-1}(1-z)^{a+b-c} w_{1}^{*} w_{2} d z=\frac{1}{a_{2} b_{2}-a_{1} b_{1}}\left[z^{c}(1-z)^{a+b-c+1}\left(w_{1}^{*} \frac{d w_{2}}{d z}-\frac{d w_{1}^{*}}{d z} w_{2}\right)\right]_{p}^{q} .
$$

From the power series $F(z)=1+\frac{a b}{c} z+\frac{a(a+1) b(b+1)}{2 c(c+1)} z^{2}+\mathcal{O}\left(z^{3}\right)$ the $z \approx 0$ asymptotic behaviour of $F, F^{\prime}, G$ and $G^{\prime}$ can be determined. Concerning the $z \approx 1$ behaviour of these functions, using $\left[\mathbb{1}\right.$ one finds $F(z) \approx k_{1} f_{1}(z), F^{\prime}(z) \approx k_{1} f_{1}^{\prime}(z), G(z) \approx k_{2} f_{2}(z)$ and $G^{\prime}(z) \approx k_{2} f_{2}^{\prime}(z)$ if $b \notin \mathbf{Z}_{0}^{-}$while $F(z) \approx k_{3} f_{2}(z), F^{\prime}(z) \approx k_{3} f_{2}^{\prime}(z), G(z) \approx k_{4} f_{1}(z)$ and $G^{\prime}(z) \approx k_{4} f_{1}^{\prime}(z)$ if $b \in \mathbf{Z}_{0}^{-}$. Here

$$
\begin{gathered}
f_{1}(z)=1-\frac{(c-a)(c-b)}{a+b-c-1}(1-z), \quad f_{2}(z)=1+\frac{a b}{a+b-c+1}(1-z), \\
k_{1}=\Gamma(c) \Gamma(a+b-c) / \Gamma(a) \Gamma(b), \quad k_{2}=-\left[k_{1}(a+b-c)\right]^{-1}, \\
k_{3}=(-1)^{|b|} \Gamma(c) \Gamma(a-c+1) / \Gamma(c-b) \Gamma(a+b-c+1), \quad k_{4}=\left[k_{3}(a+b-c)\right]^{-1}
\end{gathered}
$$

(we remark that in our case $a$ and $c$ are always positive).

By using these asymptotics we find that for $p \rightarrow 0$ (21) diverges if at least one of the corresponding $w$-s is a $G$ (except if $m=0$ and the other $w$ is an $F$, however, this case proves be of no interest) and converges if both $w$-s are $F$-s. Thus only the $F$-s are present in an orthogonal system of eigenfunctions. For $q \rightarrow 1$ the integral (21) of an $F_{1}$ and an $F_{2}$ tends to zero if $b_{1}, b_{2} \in \mathbf{Z}_{0}^{-}$, diverges if $b_{1}, b_{2} \notin \mathbf{Z}_{0}^{-}$, otherwise it tends to a nonzero finite value. Consequently, the parameter $b$ of an $F$ appearing in an orthogonal system must be a nonpositive integer. To have a maximal set of orthogonal eigenfunctions all such $b$-s have to be considered, which means $|E|=2 j+|m|, 2 j+|m|+1, \ldots$ Hence for any mass value $\kappa>0$ there exists a unique maximal orthogonal set of eigenfunctions, namely $\left\{\Psi_{m, E} \mid m=\right.$ $0, \pm 1, \pm 2, \ldots ; E= \pm(2 j+|m|), \pm(2 j+|m|+1), \ldots\}$.

We mention here that if $b$ is a nonpositive integer then $F(a, b ; c ; z)$ is nothing but the Jacobi polynomial, more precisely, $F(a, b ; c ; z)=(|b|) ! /(c)_{|b|} P_{|b|}^{(c-1, a+b-c)}(1-2 z)$ [1]. Two further remarks are that $w_{m, E}[=F(a, b ; c ; z)]$ and the corresponding $W_{m, E}$ does not depend on the sign of $m$ and $E$, and that the vanishing of the special case ( $\Psi_{m, E}, \Psi_{m,-E}$ ) is ensured not by the vanishing of the integral in (20) but by the vanishing of the constant standing before this integral [cf. the factor $\left(E_{1}+E_{2}\right)$ in (16)].

With the aid of [4], [5] and the completeness property of the Jacobi polynomials one can verify the completeness of this system of eigenfunctions.

After normalization the eigenfunctions $\Psi_{m, E}$ are of the form

$$
\begin{array}{r}
\Psi_{m, E}(t, r, \vartheta)=\frac{(-i)^{|m|}}{\sqrt{2}}\left(\begin{array}{c}
1+\frac{E}{\kappa} \\
1-\frac{E}{\kappa}
\end{array}\right) \sqrt{\frac{\kappa}{2 \pi} \frac{\Gamma(a) \Gamma(c-b)}{\Gamma(c)^{2} \Gamma(a-c+1) \Gamma(1-b)}} \times \\
z^{\frac{c-1}{2}}(1-z)^{\frac{a+b-c+1}{2}} F(a, b ; c ; z) e^{i m \vartheta} e^{-i E t}
\end{array}
$$


where $z=\tanh ^{2}(r)$ - the complex phase factor $(-i)^{|m|}$ is introduced for later convenience. Concerning our identification $(t=2 \pi) \equiv(t=0)$ we can observe that if $2 j$ is not an integer value then a-space-, $m$ - and $E$-independent, hence fortunately harmless (see Sec. VII) - phase factor appears between $\Psi_{m, E}(t=2 \pi)$ and $\Psi_{m, E}(t=0)$.

Similarly to the Minkowski case, the energy eigenstates with positive eigenvalue prove to be 'positive' (see Sec. III) and those with negative eigenvalue are 'negative'. The positive wave functions are the particle states, and the negative ones are the antiparticle states. Here we can see how naturally the antiparticle modes appear in our approach.

Now let us introduce the notation $\left|k_{L}, k_{R}\right\rangle$ for the eigenstate (23) where $k_{L}=$ $(E-m) / 2, k_{R}=(E+m) / 2$. The possible values of $k_{L}$ and $k_{R}$ are such that $\left|k_{L}\right|,\left|k_{R}\right|=j, j+1, j+2, \ldots$ and for positive eigenstates both $k_{L}$ and $k_{R}$ are positive while for a negative state both are negative. With these notations

$$
H\left|k_{L}, k_{R}\right\rangle=\left(k_{L}+k_{R}\right)\left|k_{L}, k_{R}\right\rangle \quad \text { and } \quad J\left|k_{L}, k_{R}\right\rangle=\left(k_{R}-k_{L}\right)\left|k_{L}, k_{R}\right\rangle .
$$

\section{Symmetry properties}

Comparing our results with [1] (see Sec. II) we can see that our investigation reproduces the findings of [1], while it gives account of the antiparticle states and the topologically nontrivial cases $j \notin\left\{\frac{3}{2}, \frac{4}{2}, \ldots\right\}$ as well. (The quantum numbers $k_{L}, k_{R}$ provide a bit more convenient possibility for a common labelling of the positive and negative eigenstates, this is the reason why we shifted $n_{L}$ and $n_{R}$ to $k_{L}$ and $k_{R}$ by an appropriate $\pm j$.) Also, the choice of a timelike vector $K$ in [1] corresponds here to a choice of a middle-inertial reference frame based on an absolute velocity value $U$. What is left is to verify the symmetry properties of the energy eigenstates in our approach, and to discuss why in [四] only the topologically trivial cases are found.

The left and right translations $g \mapsto f g, g \mapsto g \tilde{f}^{-1}$ naturally lead to the representations $\left[D_{l}(f) \psi\right](g)=\psi\left(f^{-1} g\right),\left[D_{r}(\tilde{f}) \psi\right](g)=\psi(g \tilde{f})$ on a $\psi: S L(2, \mathbf{R}) \rightarrow \mathbf{C}$. The corresponding infinitesimal generators, which give a representation of the Lie algebra elements $U, X$ and $Y$, are

$$
\begin{aligned}
l_{U} & =-\partial_{\vartheta}-\partial_{t} \\
l_{X} & =-\cos (\vartheta+t) \partial_{r}+\operatorname{coth} r \sin (\vartheta+t) \partial_{\vartheta}+\tanh r \sin (\vartheta+t) \partial_{t}, \\
l_{Y} & =-\sin (\vartheta+t) \partial_{r}-\operatorname{coth} r \cos (\vartheta+t) \partial_{\vartheta}-\tanh r \cos (\vartheta+t) \partial_{t}, \\
r_{U} & =-\partial_{\vartheta}+\partial_{t} \\
r_{X} & =\cos (\vartheta-t) \partial_{r}-\operatorname{coth} r \sin (\vartheta-t) \partial_{\vartheta}+\tanh r \sin (\vartheta-t) \partial_{t}, \\
r_{Y} & =\sin (\vartheta-t) \partial_{r}+\operatorname{coth} r \cos (\vartheta-t) \partial_{\vartheta}-\tanh r \cos (\vartheta-t) \partial_{t}
\end{aligned}
$$

The transformations $D_{l}(f), D_{r}(\tilde{f})$ are symmetries of the system, i.e., they transform 
a solution of (12) to another solution of it, as can be verified by means of the infinitesimal generators.

We are interested in the representation of the left and right translations on the $\Psi$-s, which can be obtained from $D_{l}$ and $D_{r}$ using the relation between a $\psi$ and the corresponding $\Psi$ [cf. (17)]:

$$
D_{L}(f) \Psi=\frac{1}{\sqrt{2}}\left(\begin{array}{c}
D_{l}(f) \psi+\frac{i}{\kappa} \partial_{t}\left[D_{l}(f) \psi\right] \\
D_{l}(f) \psi-\frac{i}{\kappa} \partial_{t}\left[D_{l}(f) \psi\right]
\end{array}\right)
$$

and the analogous formula for $D_{R}(\tilde{f})$. The action of the infinitesimal generators of $D_{L}$ and $D_{R}$ on a $\Psi$ can be written as

$$
L_{k}\left(\begin{array}{c}
\varphi \\
\chi
\end{array}\right)=\left(\begin{array}{c}
l_{k} \varphi+\frac{i}{2 \kappa}\left[\partial_{t}, l_{k}\right](\varphi+\chi) \\
l_{k} \varphi-\frac{i}{2 \kappa}\left[\partial_{t}, l_{k}\right](\varphi+\chi)
\end{array}\right)
$$

( $k$ stands for $U, X, Y$ ) and similarly for $R_{k}$. As one can check, the $L_{k}$-s and $R_{k^{-S}}$ satisfy the same commutation relations as the $l_{k}$-s and $r_{k}$-s. The operators $L_{0}=\frac{1}{i} L_{U}$, $L_{1}=\frac{1}{i} L_{X}$ and $L_{2}=\frac{1}{i} L_{Y}$ satisfy (3). The action of $L_{0}$ and $L_{ \pm}=L_{1} \mp i L_{2}$ on an energy eigenstate can be determined by a straightforward if lengthy calculation involving [4], the result is

$$
\begin{aligned}
L_{0}\left|k_{L}, k_{R}\right\rangle & =2 k_{L}\left|k_{L}, k_{R}\right\rangle, \\
L_{+}\left|k_{L}, k_{R}\right\rangle & =2 \sqrt{\left(k_{L}+j\right)\left(k_{L}+1-j\right)}\left|k_{L}+1, k_{R}\right\rangle, \\
L_{-}\left|k_{L}, k_{R}\right\rangle & =2 \sqrt{\left(k_{L}-1+j\right)\left(k_{L}-j\right)}\left|k_{L}-1, k_{R}\right\rangle .
\end{aligned}
$$

With the analogously defined operators $R_{0}, R_{1}, R_{2}$ and $R_{ \pm}$one finds

$$
\begin{aligned}
R_{0}\left|k_{L}, k_{R}\right\rangle & =-2 k_{R}\left|k_{L}, k_{R}\right\rangle, \\
R_{-}\left|k_{L}, k_{R}\right\rangle & =-2 \sqrt{\left(k_{R}+j\right)\left(k_{R}+1-j\right)}\left|k_{L}, k_{R}+1\right\rangle, \\
R_{+}\left|k_{L}, k_{R}\right\rangle & =-2 \sqrt{\left(k_{R}-1+j\right)\left(k_{R}-j\right)}\left|k_{L}, k_{R}-1\right\rangle .
\end{aligned}
$$

The linear subspaces spanned by the positive, resp. the negative, energy eigenstates are invariant subspaces of the left and right transformations. Hence both the left and the right representations are a direct sum of two irreducible representations, similarly to what happens in the case of Minkowski spacetime. For the sake of simplicity, in the following we will consider the positive subspace and the left translations only, the parallel discussion of the negative subspace and/or for the right translations is straightforward.

If $j \in\left\{\frac{3}{2}, \frac{4}{2}, \ldots\right\}$ then the eigenvalues of $L_{0}$ are integers so the operators $e^{i \tau L_{0}}=$ $e^{\tau L_{U}}$ give a continous representation of the one-parameter subgroup $e^{\tau U}$ of $S L(2, \mathbf{R})$. Thus the identification of $\tau=0$ and $\tau=2 \pi$ does not give any problem. For other values of $j e^{\tau L_{U}}$ is not continous in $\tau=0 \equiv 2 \pi$. Hence in these cases the representation (28) of the algebra $\operatorname{sl}(2, \mathbf{R})$ cannot be exponentiated to a continous 
representation of the group $S L(2, \mathbf{R})$. However, the eigenvalues of the operator $\tilde{L}_{0}=$ $L_{0}-\{2 j\}$ are integers $(\{2 j\}$ denotes the fractional part of $2 j)$. Correspondingly, for arbitrary $j$-s, let us introduce the mapping $\tilde{D}_{L}^{j}$ that maps a group element $e^{\xi X} e^{\eta Y} e^{\tau U}$ to the operator $e^{\xi L_{X}} e^{\eta L_{Y}} e^{i \tau \tilde{L}_{0}}=e^{\xi L_{X}} e^{\eta L_{Y}} e^{\tau L_{U}} e^{-i \tau\{2 j\}}$. In the special cases $j=$ $\frac{3}{2}, \frac{4}{2}, \ldots$ this $\tilde{D}_{L}^{j}$ gives the representation $D_{L}$, and for the other values of $j \tilde{D}_{L}^{j}$ is a ray representation. The infinitesimal generators of this ray representation, $\tilde{L}_{X}=L_{X}$, $\tilde{L}_{Y}=L_{Y}$ and $\tilde{L}_{U}=i \tilde{L}_{0}$, form a central extension of the Lie algebra $\operatorname{sl}(2, \mathbf{R})$ :

$$
\left[\tilde{L}_{U}, \tilde{L}_{X}\right]=2 \tilde{L}_{Y}, \quad\left[\tilde{L}_{X}, \tilde{L}_{Y}\right]=-2 \tilde{L}_{U}-2\{2 j\} i, \quad\left[\tilde{L}_{Y}, \tilde{L}_{U}\right]=2 \tilde{L}_{X},
$$

the constant $2\{2 j\}$ plays the role of the commutator cocycle. (To prove that $\tilde{D}_{L}^{j}$ is indeed a ray representation it is enough to verify that (30) fulfils the conditions for a central extension, see [6].) We can see that, by carrying out the shift $\tilde{L}_{X} \rightarrow$ $L_{X}, \tilde{L}_{Y} \rightarrow L_{Y}, \tilde{L}_{U} \rightarrow L_{U}$ on the central extension, we arrive at a representation of the algebra $\operatorname{sl}(2, \mathbf{R})$. Conversely, starting from an arbitrary unitary irreducible representation of $s l(2, \mathbf{R})$ (indexed by a $j>1$ ), an appropriate redefinition yields such infinitesimal operators that can be exponentiated to a continous unitary ray representation of the left translation symmetry. In the cases $j=\frac{3}{2}, \frac{4}{2}, \ldots$ this ray representation is indeed a representation.

The situation corresponds to the following general picture. A symmetry group is represented on the Hilbert space of a quantum theory, in general, by a continous unitary ray representation. The infinitesimal generators of the ray representation give a central extension of the Lie algebra of the group. If the symmetry group is semi-simple then the commutator cocycle can be transformed out by an appropriate shift of the infinitesimal generators, the new infinitesimal operators satisfy the commutation relations of the Lie algebra with no central elements. Thus we arrive at a unitary representation of the Lie algebra.

Hence we can see that in [1] only those cases are presented where a representation of the symmetry group occurs, the ray representation cases are not found. The reason of this is that in [1] only those irreducible unitary representations of $\operatorname{sl}(2, \mathbf{R})$ are considered, which provide a representation of $S L(2, \mathbf{R})$ as well. The analysis above shows that the other $\operatorname{sl}(2, \mathbf{R})$ representations are also relevant in our physical system.

To make our considerations complete, in the end let us verify that the phase factor appearing between $\Psi_{m, E}(t=2 \pi)$ and $\Psi_{m, E}(t=0)$ in the ray representation cases does not lead to any physical inconsistency. Fortunately, this phase factor is space-, $m$ - and $E$-independent, thus it is simply an overall constant phase factor for all the wave functions. It causes a discontinuity of the wave functions only in the time variable $[$ at $(t=2 \pi) \equiv(t=0)]$, while the inner product involves integration over the space variables (cf. Sec. V), and observables, such as the momentum or the angular momentum, include derivations with respect to the space variables. A constant phase factor does not change the expectation value of an observable or the inner product of two wave functions, thus an expectation value or an inner product calculated at $t=0$ is the same as at $t=2 \pi$. So we can see that the physically 
observable quantities allow the identification $(t=2 \pi) \equiv(t=0)$, the presence of the phase factor does not disturb the physical interpretation issues and leads to no inconsistency.

Acknowledgements: The author wishes to thank L. Palla, Z. Horváth, L. Szabados and G. Takács for valuable discussions.

\section{References}

[1] G. Jorjadze, L. O’Raifeartaigh and I. Tsutsui, "Quantization of a relativistic particle on the $S L(2, \mathbf{R})$ manifold based on Hamiltonian reduction", Phys. Lett. B 336 (1994) 388.

[2] V. Bergmann, Ann. Math. 48 (1947) 568.

[3] H. Feshbach and F. Villars, "Elementary Relativistic Wave Mechanics of Spin 0 and Spin 1/2 Particles", Rev. Mod. Phys. 30 (1958) 24.

[4] "Handbook of Mathematical Functions" (M. Abramowitz and I. Stegun, Ed.), Nauka, Moscow, 1979, in Russian; especially formulae 15.1.20, 15.2.1, 15.2.10$27,15.3 .3,15.3 .6-12,15.4 .6$ and 22.12.1.

[5] A. ERdélyi ET.AL., "Higher Transcendental Functions", McGraw-Hill, New York, 1953.

[6] P. Goddard and D. Olive, Int. J. Mod. Phys. A, Vol. 1, No. 2 (1986) 303; T. Matolcsi And S. SzÉKely, "Mathematical Physics I.", Tankönyvkiadó, Budapest, 1980; in Hungarian.

[7] "Handbook of Applied Mathematics" (C.E. Pearson, Ed.), Van Nostrand Reinhold, New York, 1990. 